\title{
Experiments with Cooperative Control of Underwater Robots
}

\author{
Matthew Dunbabin* Iuliu Vasilescu ${ }^{\dagger}$ Peter Corke* Daniela Rus ${ }^{\dagger}$ \\ * CSIRO ICT Centre, Brisbane, Australia. \\ $\dagger$ Massachusetts Institute of Technology, Cambridge, Massachusetts, USA.
}

August 18, 2008

\begin{abstract}
In this paper we describe cooperative control algorithms for robots and sensor nodes in an underwater environment. Cooperative navigation is defined as the ability of a coupled system of autonomous robots to pool their resources to achieve long-distance navigation and a larger controllability space. Other types of useful cooperation in underwater environments include: exchange of information such as data download and retasking; cooperative localization and tracking; and physical connection (docking) for tasks such as deployment of underwater sensor networks, collection of nodes, and rescue of damaged robots. We present experimental results obtained with an underwater system that consists of two very different robots and a number of sensor network modules. We present the hardware and software architecture of this underwater system. We then describe various interactions between the robots and sensor nodes and between the two robots, including cooperative navigation. Finally, we describe our experiments with this underwater system and present data.
\end{abstract}

\section{Introduction}

The application of autonomous mobile networks of robots and sensors to the underwater domain has great potential for monitoring the health of river and marine environments. The oceans alone cover $70 \%$ of our planet and along with rivers and lakes are critical to our well-being. Monitoring these environments is difficult and costly for humans: divers are regulated in the hours and depths at which they can work, and they require a boat on the surface that is costly to operate and subject to weather conditions. A networked system of robots and sensors deployed underwater could monitor physical variables such as water temperature and pressure as well as variables such as conductivity, turbidity and certain pollutants. The network could, for example, track plumes of pollutants or silt due to dredging operations. It could monitor the behavior of underwater ecosystems, and model their response to weather and human activities, or it could perform surveillance of ports or underwater geochemical prospecting. Each of these applications requires long term underwater presence over a large area and adaptation to changes in the environment, by positioning and repositioning the sensors and robots or adjusting the sampling rate.

We wish to develop a system of cooperative underwater robots and sensors that are networked together for versatile cooperative tasks such as deployment, sensor localization, system placement and maintenance of desired network properties, leading to self-organized underwater systems. The system travels together to the mission site. Once at the destination, the system disassembles into task-specific components. We believe that this goal an be achieved by the simultaneous investigation of three research thrusts: (1) developing experimental devices in support of cooperative networked underwater tasks; (2) developing control and planning algorithms for networked coupled cooperative underwater systems; and (3) implementing the proposed algorithms and deploying them in ocean environments.

In this paper we describe our results with cooperative control of coupled underwater robots. Specifically, the key technical contributions of this work are (1) a system architecture that support cooperative control, 
(2) the cooperative navigation algorithm, (3) the application of this algorithm to data muling, and (4) the experimental characterization of cooperative navigation.

Cooperation is an important aspect of designing useful underwater robots. Long-range and long-endurance underwater operations require substantial power and thus a large robot. Once at the destination the large size may be a disadvantage if the task requires high maneuverability or a small size, for example, to enter a wreck. We would like to have robot systems that meet both needs: (1) they can perform long-range travel while also (2) being maneuverable at the destination. We propose cooperation as a means for accomplishing this.

One approach to realizing these two goals is to create heterogeneous robots that will cooperate in long distance travel by docking together and taking advantage of the increased number of resources (e.g. thrusters and power) in the system. Once at the destination, they can disassemble into, or unload, smaller and more maneuverable and perhaps task-specific robots that work individually or in concert. Many tasks require localization and this could be achieved by a cooperative system of robots that self-deploy in a way that forms a networked system for high-precision distributed localization and tracking [Moore et al., 2004, Detweiler et al., 2006]. Finally, such cooperating systems could sustain long-term operation by designating one robot as a power and recharging station.

An alternative approach is modular cooperative underwater robots that can deploy and recover sensor networks. The deployer is an autonomous vehicle with stackable sensing modules of uniform shape. Each module has a computation, motor, buoyancy, and battery module, and an arbitrary number of sensor modules. A docking mechanism allows a module to attach to the one above it in the structure. A fleet of such robots can be controlled to place sensors at designated locations on pipelines, pillars, or specific locations at the bottom of the ocean. Once in place, each sensor collects data, assists with monitoring tasks, and participates in guiding the AUVs. The robots can also be used to reposition the network to repair and maintain network connectivity (similar to the ideas in [Corke et al., 2004a, Corke et al., 2004b]). The multi-module nature of the system coupled with its ability to dock to arbitrary units also provides redundancy: when a robot is damaged, a different robot can locate it, dock with it, and retrieve it.

More specifically, in this paper we build on our work in [Dunbabin et al., 2006b] and describe our first steps toward the vision of cooperative modular underwater robots. We present a heterogeneous system that consists of two robots with very different capabilities and a collection of static underwater sensor nodes, and all system elements are networked acoustically and optically. When coupled together, the robots can travel further, faster, and along a larger set of trajectories than each individual robot. Because the robots have different types of perception and communication resources, the coupled system can also execute more complex tasks than either robot alone. We focus on the algorithmic and systems issues related to enabling these robots to become physically coupled and to coordinate navigation by sharing resources (thrusters and sensors). A docking mechanism and algorithm enables the two robots to become physically coupled. We describe in detail how we achieve navigation control and present experimental data from several trials in a test tank. We describe how one robot is able to rescue another, and finally, we describe an application of the cooperative system to data muling from an in-situ sensor network. Much work remains to be done to achieve the full potential of cooperative underwater robotics. We have encouraging initial steps in this direction.

\subsection{Related Work}

There has been much work in the fields of Autonomous Underwater Vehicles (AUVs), their control and navigation, underwater communications, sensors and docking [Yuh, 2000,Park et al., 2007,Pizarro et al., 2004, Walter et al., 2007]. In a 2000 survey [Whitcomb, 2000], it was estimated that over 1,000 robotic underwater vehicles operate worldwide in industry, military and research applications. A small but growing portion of these are semi-autonomous or autonomous robots. AUVs in general face severe navigation challenges due to the fact that water absorbs radio waves. There have been three types of navigation systems for autonomous robots underwater, which rely on different sensors: (1) dead-reckoning and inertial navigation, (2) acoustic, and (3) geophysical navigation [Leonard et al., 1998]. Prior work includes visual feature tracking [Wettergreen et al., 1999], visual SLAM [Walter et al., 2007], large-scale mosaicing [Pizarro et al., 2004], and visual and inertial fusion [Huster and Rock, 2003, Dunbabin et al., 2004]. 
Others have found that combining sensor information, such as conventional long baseline acoustic sensing and Doppler effect, can improve underwater navigation [Whitcomb et al., 1999]. Optical guidance systems, which give precise resolution at very short distances, have been used for the AUV's close-range homing and docking abilities [Cowen et al., 1997, Park et al., 2007, Bellingham et al., 1994]. Alternatively, long baseline and ultra-short baseline acoustic beacons have also been used for docking [Singh et al., 1997].

Recently there has been interest in deploying multiple robots, in particular for oceanographic research applications. The Serafina project [Kalantar and Zimmer, 2004] explores large-scale formation control issues with multiple small, agile AUVs. Gliders such as Seaglider [Eriksen et al., 2001] are designed to dive to a programmed depth and resurface while taking measurements, moving for thousands of kilometers in a vertical sawtooth pattern that uses very little power. Gliders have also been used in cooperative multi-AUV control research [Fiorelli et al., 2004]. It is becoming more important for the robots to be able to assist in the deployment or to act as parts of such large-scale data-collecting networks. A small submarine [Bokser et al., 2004] has been proposed as a sensor in such a network. The robot houses a Mote sensor and can control its own depth. In addition, some attempts have been made to create modular underwater robots. Inspired by eels [McIsaac and Ostrowski, 1999] or lampreys [Ayers et al., 2000], these are smaller-scale biomimetic robots whose modules are permanently joined in one configuration.

An important aspect of cooperative underwater systems is realizing adaptive control. The ODIN project [Choi et al., 2003, Hanai et al., 2004] aims to create underwater robots with redundant thrusters that can learn their body models and synthesis controllers matching the learned models.

\subsection{Outline}

This paper is organized as follows. Section 2 presents the heterogeneous hardware used in our work. Section 3 describes the cooperative behaviours, in particular the algorithms for cooperative navigation and presents our experimental results. Finally, Section 4 shows the application of cooperative navigation and resource pooling by the two robots to data muling over an underwater sensor network.

\section{System Description}

The cooperative underwater robot systems that we envisage are predicated on physical mechanisms to dynamically and automatically locate and dock with other modules. Creating such underwater robot systems poses many engineering challenges. These challenges are centered around designing the basic underwater robot, its perception, navigation, and communication system, and the inter-module connection mechanism. We have developed a heterogeneous underwater system that consists of two different robots and a collection of static underwater sensor nodes, see Figure 1. The robots and the sensor nodes are designed to be capable of docking with each other. All the modules in the system can also communicate as an ad-hoc network using acoustic communications. They can also communicate locally using optical communication. Finally, they can communicate when docked via the docking mechanism. This section describes in more detail the components of this system: the underwater sensor network nodes called AquaFlecks and two different types of Autonomous Underwater Vehicles (AUVs) called Starbug and Amour. The hardware and networking infrastructure of this system has been described in detail in [Vasilescu et al., 2005].

\subsection{AquaFlecks}

AquaFlecks are underwater sensor network nodes that package together computation, acoustic and optical communication, and a suite of sensors in a $170 \times 100 \times 90$ mmyellow water-tight enclosure.

The box contains a wireless sensor network node [Sikka et al., 2004] based on an 8-bit processor with $512 \mathrm{kbyte}$ of flash memory for data logging/storage. Each node has a pressure sensor, temperature sensor, and a CMUCam camera capable of taking $255 \times 143$ pixel color pictures. Optical and acoustic communications systems are described in more detail in Section 2.4. Three alkaline C-cells provide $27 \mathrm{~W}$-h which allows four 


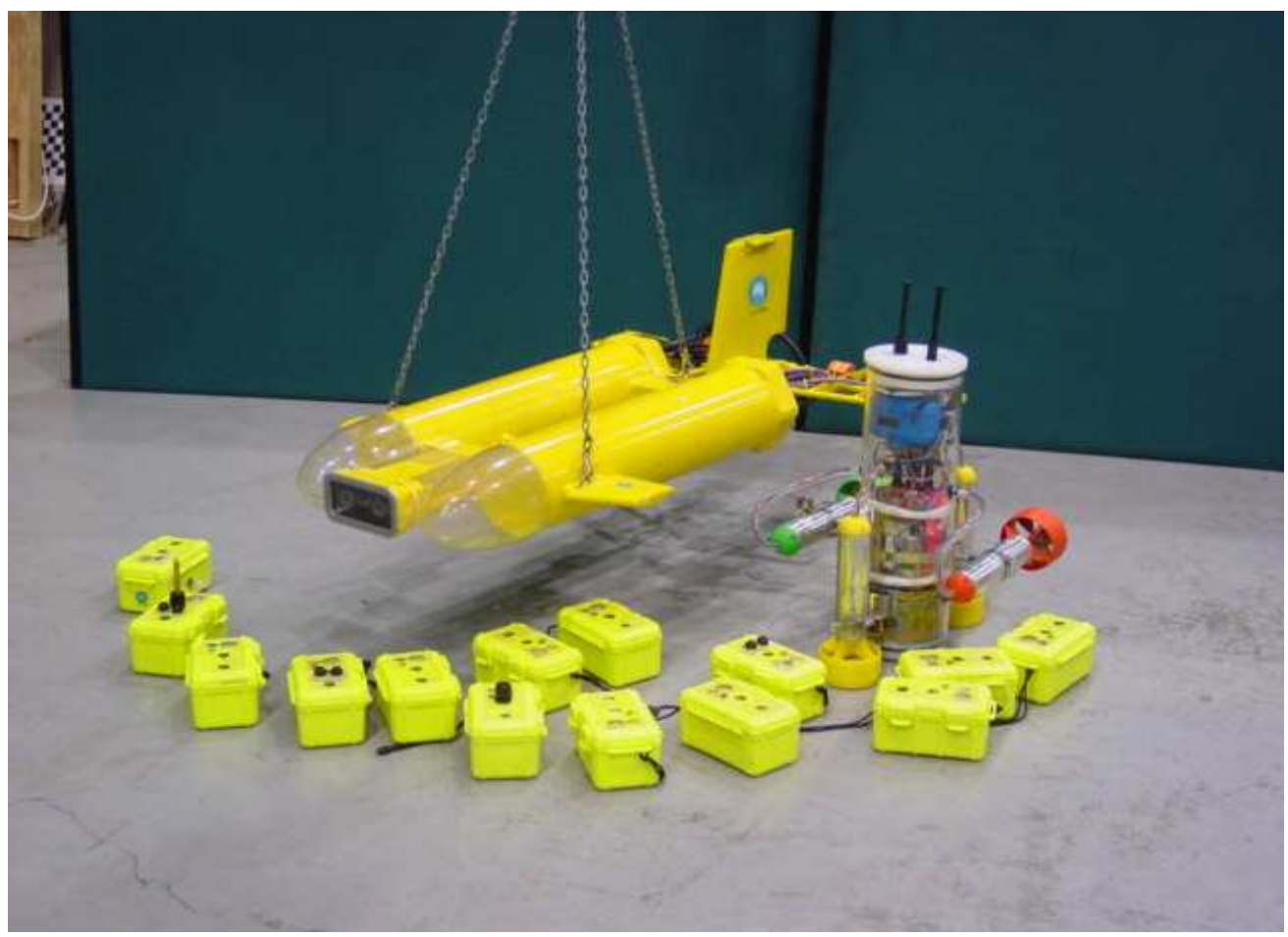

Figure 1: The underwater robot and sensor family. Back row from the left: Starbug AUV then Amour AUV. Front row the AquaFleck underwater sensor nodes.

days of continuous operation with all sensors and communication hardware fully powered - duty cycling would greatly extend the endurance.

The top side of the box supports a docking rod which allows the node to be picked up and carried. An LED beacon at the top aids the docking process. The docking system is described further in Section 3.1.

\subsection{Amour AUV}

Amour, shown in Figure 3, is an AUV developed at MIT which is equipped with powerful thrusters and an integral docking mechanism that allows it to find and mate with the AquaFleck. Due to its docking capabilities and the high power-to-weight ratio, in this study the Amour AUV acts as the rescuer and "work-horse" providing the capability to find, dock and cooperatively move both robots.

Amour has on-board computation, data storage, batteries, as well as acoustic and optical communication that allow it to communicate with an AquaFleck. The bottom cap of the robot has a cone shaped cavity for the docking mechanism.

Amour's key performance specifications are: mass $(11 \mathrm{~kg})$, length $43.3 \mathrm{~cm}$, diameter $15.3 \mathrm{~cm}$, maximum linear speed $1 \mathrm{~m} / \mathrm{s}$, maximum rotation speed $360 \mathrm{deg} / \mathrm{s}$. Each of the four external thrusters has a maximum power of $150 \mathrm{~W}$ and a maximum static thrust of $35 \mathrm{~N}$. Two thrusters act vertically and the other two act horizontally to provide forward-backward propulsion and yaw control (see Figure 3). Power is supplied by a 140W-h lithium-polymer battery which gives an endurance of 3 hours.

The robot's body consists of a clear acrylic tube. The main processor is a 8-bit microcontroller with $64 \mathrm{kbyte}$ of program memory and $2 \mathrm{kbyte}$ of RAM and the main navigation sensors are pressure and magnetic compass. 


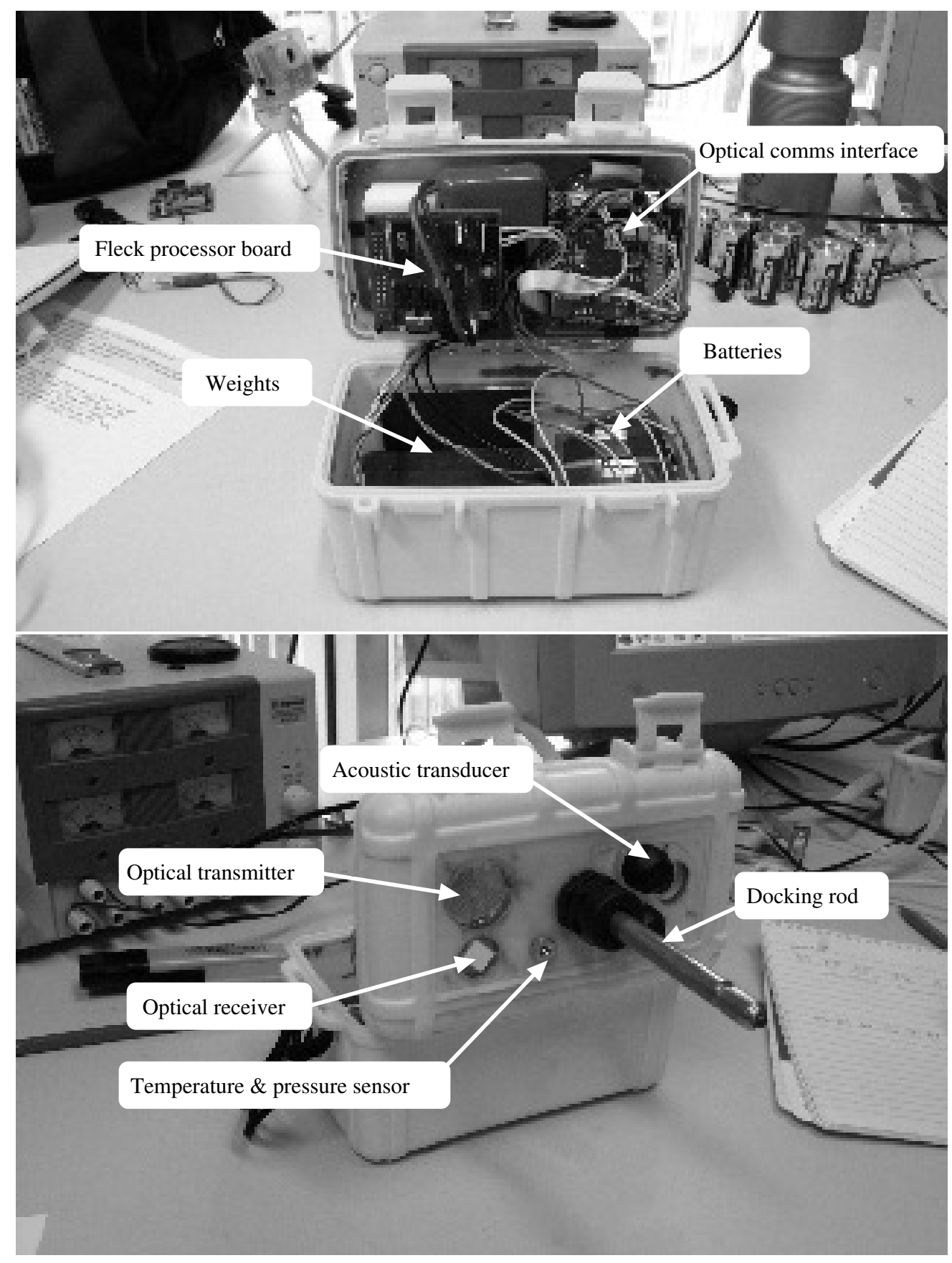

Figure 2: The AquaFleck underwater sensor node.

\subsection{Starbug AUV}

Starbug, shown in Figure 4, is a hybrid AUV developed at CSIRO for shallow water photographic and instrument survey [Dunbabin et al., 2005]. Its key performance specifications are: mass $26 \mathrm{~kg}$, length $1.2 \mathrm{~m}$, maximum forward thrust $20 \mathrm{~N}$, maximum speed $1.5 \mathrm{~m} / \mathrm{s}$, and maximum endurance of 3.5 hours $(8 \mathrm{~km}$ at 


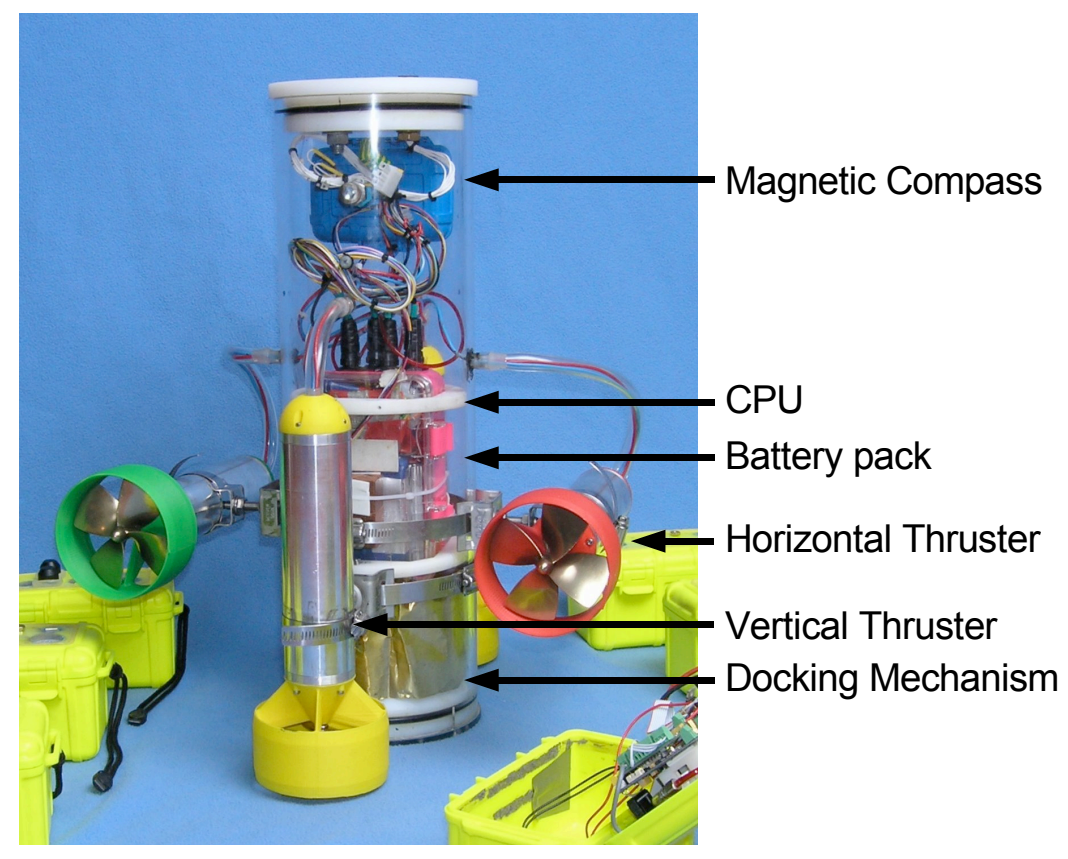

Figure 3: The Amour AUV.

$0.7 \mathrm{~m} / \mathrm{s}$ ) with a $250 \mathrm{~W}-\mathrm{h}$ lead-acid battery. In the configuration used in this study the vehicle is underactuated with only five thrusters, providing forward, vertical, yaw, roll and pitch control, but not lateral force.

The on-board sensing capabilities used in this study are roll, pitch, yaw, depth and the stereo camera system. The AUV has two stereo vision heads, one looking downward for sea-floor altitude and odometry estimation as well as mapping, and the other looking forward for obstacle avoidance. The CMOS cameras have a baseline of $70 \mathrm{~mm}$ and an on-board dual stereo MUX board provided two $320 \times 288$ pixel interlaced images to the CPU. All image processing and vehicle control was performed on an $800 \mathrm{MHz}$ Crusoe processor stack running Fedora Linux. A single CANbus links all the thrusters. The visual odometry algorithm employed in this series of experiments, detailed in [Dunbabin et al., 2005], has proven effective in over $150 \mathrm{~km}$ of ocean surveys and demonstrates real-time position estimation performance better than $5 \%$ of distance travelled. In this work the downward cameras are also used to recognize AquaFleck nodes using color segmentation.

\subsection{Communications Hardware}

Our approach to communication is based on a hybrid design which incorporates both optical and acoustic systems. The optical system is used for short-range line-of-sight data transfer and communication between a sensor node and an AUV. One application is for the AUV to move through the network uploading stored data from the sensor nodes, and downloading commands. The acoustic system is used to signal events and transmit small amounts of data. Signalling an event allows the AUV to move to the area of interest, and may trigger a redeployment of the sensor network to concentrate on some important feature in the environment. The signal could also be used by the AUV to request a node to emit an acoustic ping or illuminate its docking beacon.

The optical transmitter uses a high power, $700 \mathrm{~mW}$, green LED chosen based on the sensitivity of the photodiode and the attenuation of light as described in [Vasilescu et al., 2005]. The data rate is $320 \mathrm{kbit} / \mathrm{sec}$ and the supporting protocols are also described in [Vasilescu et al., 2005]. The protocol allows an AquaFleck 


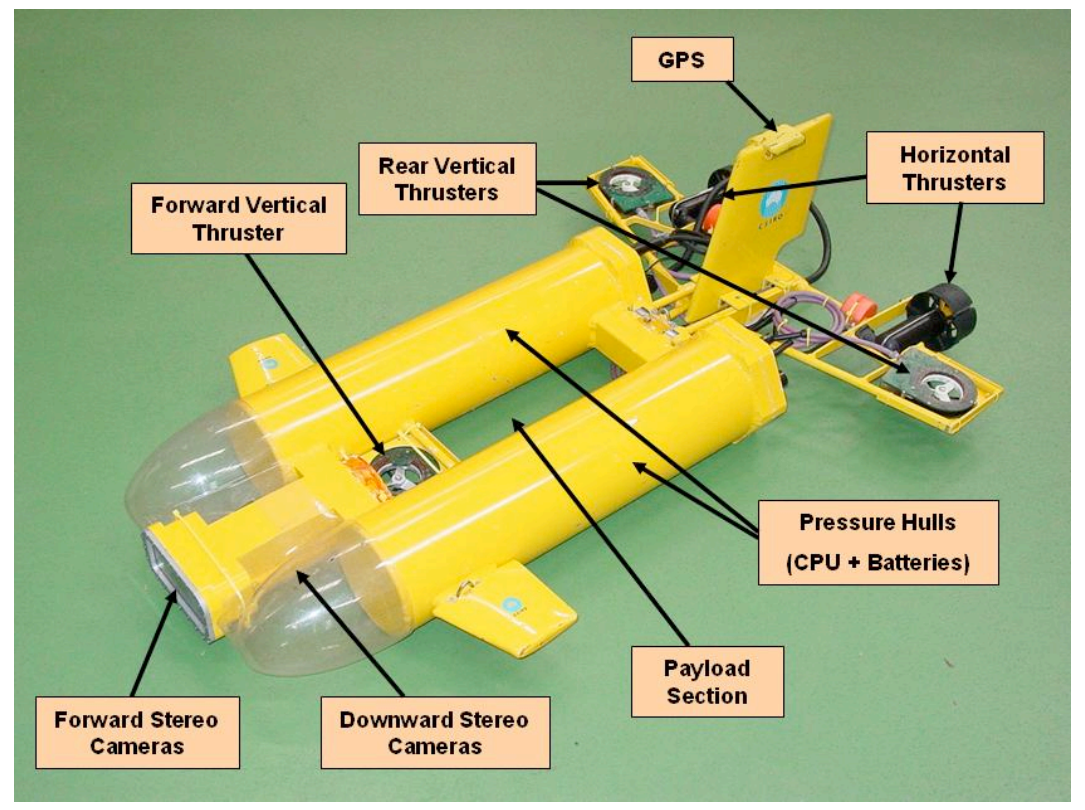

Figure 4: The Starbug Mk I AUV. The docking AquaFleck is secured in the payload section at approximately the vehicle's centre of gravity. The down stereo camera pair (obscured from view) is in the front camera housing. Longitudinal and yaw control performed by main thrusters, with depth, roll and pitch performed by three flat thrusters in the horizontal plane ( 1 forward and 2 aft).

to be interrogated for its identity (and implicit in this is the location of the node), its capability (what sensors it carries) and its data availability (how much data it has to download). A simple state-less protocol is used to download data one block at a time and to free the storage on board the AquaFleck. The communication range in clear water is $8 \mathrm{~m}$ with a cone angle of 30 degrees.

The acoustic transducers employed on the nodes are normally used for acoustic transmission in air. Rated as "weatherproof" and completely sealed they can be used in water. Nominally operating at $40 \mathrm{kHz}$ in air, when driven at $30-32 \mathrm{kHz}$ the acoustic coupling with water is very good and they emit uniformly over a hemisphere with a maximum range of $25 \mathrm{~m}$. We have since developed a second generation acoustic networking system capable of ranging which is described in [Corke et al., 2007].

\subsection{Docking Hardware}

On the underside of the Amour AUV is cone shaped cavity which allows docking/mating with any structure that holds a docking element - a $15.3 \mathrm{~cm}$ long rod of $1 \mathrm{~cm}$ diameter with a modulated optical docking beacon at its base. The alignment during docking is aided by the probe and drogue configuration (similar to the NASA Apollo docking mechanism) of the connecting sides, as seen in Figure 5. The conical bottom cap of the robot guides the rod into place during docking.

At the apex of the cone a latch plate with a variable width hole moves freely in the horizontal plane in a tub-like compartment. The plate is attached on one side to a permanent magnet. An electromagnet is mounted coaxially with the permanent magnet behind a thin waterproof wall. When the robot is ready to dock, a current is sent to the electromagnet which repels the magnetic latch. The larger diameter hole is thus positioned above the apex of the cone. The probe can then enter and is latched in place by temporarily reversing the polarity of the electromagnet to boost the attraction between the magnets. The attractive force at rest is enough to keep the probe latched. Once docked the latching mechanism can hold the elements together with up to $200 \mathrm{~N}$ of force. 

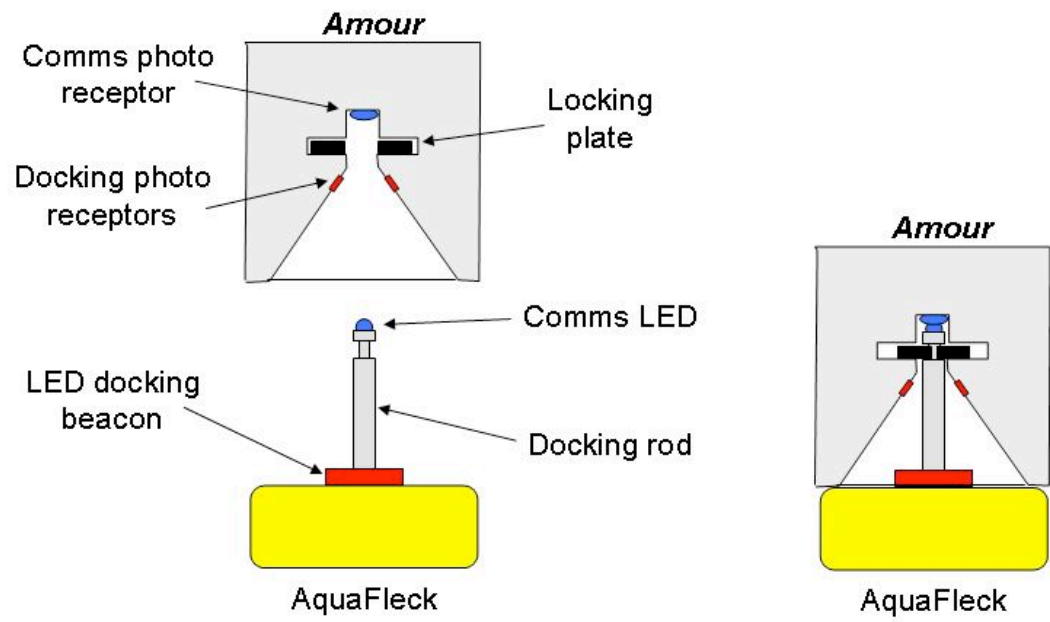

Figure 5: Schematic of Amour/AquaFleck docking system. (left) Key parts labelled prior to docking, (right) configuration after docking (Note the locking pins clasp the AquaFleck docking rod).
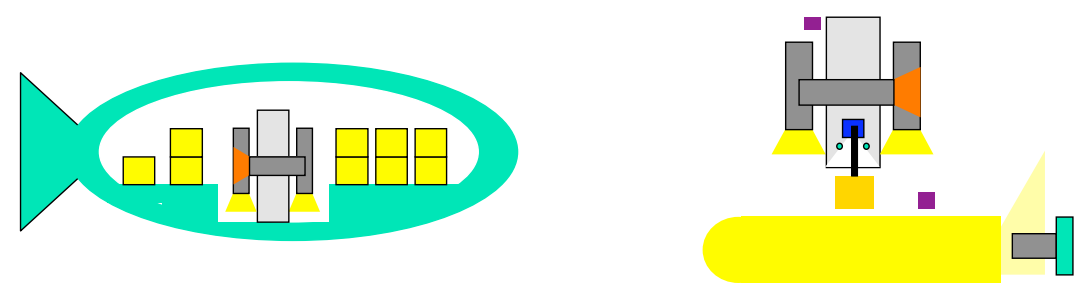

Figure 6: (Left) Cooperative navigation in an ideal underwater system. (Right) Cooperative navigation with Amour and Starbug.

An LED docking beacon located at the base of the docking rod provides the primary guidance during the docking process. The LED is modulated at high frequency so that its signal can be uniquely recognized despite interference from ambient light. The conical cavity contains four PIN-diode light sensors pointing in complementary directions which can determine the direction of the LED docking beacon up to $8 \mathrm{~m}$ away in clear water.

The magnetic latching mechanism is first triggered when the photodiode array suddenly stops sensing light after returning maximum values for a short period of time. This indicates that the probe has entered the drogue. At that time, the single photodiode mounted above the variable-width hole in the latching plate will sense the light emitted from the communications LED on the tip of the rod.

Another LED at the tip of the docking rod provides one-way communications from the element with the docking rod to the element with the docking cone, once they are docked. This is an additional communications path to those described in Section 2.4.

\section{Cooperative Navigation}

Figure 6 shows the general idea of cooperative navigation. By packaging together the modules of the underwater system we can achieve longer distances traveled. The system we have built achieves the notion of physical coupling for long distance navigation and pooling of resources.

In this section we describe the algorithms that enable two autonomous underwater robots to dock. We 
then discuss and analyze the algorithm for cooperative navigation. We believe that this capability is useful for a variety of real-world applications. Some are relatively simple such as picking up a robot or sensor node, moving to a location and placing a node. Similarly the robot could collect nodes from known locations. The critical capability is for docking and undocking. Using these same capabilities Amour can pick up Starbug from the pool floor, showing how it is possible for one robot to rescue another. We also require the capability for control of a coupled robot system, which will be over actuated and have significantly different dynamic properties to each robot individually.

The scenarios described are based on the capabilities of the robots used in this work. Due to Starbug's greater environmental perception capabilities, in this study it acts as the "leader" or "master" when docked with Amour, providing the sensor processing facilities, closed-loop control of both itself and Amour, as well as mission planning and execution. Docking is achieved by an AquaFleck physically attached to Starbug and sitting in its payload bay with its docking rod upward. This AquaFleck was modified such that it accepted an RS232 connection to Starbug's on board computer. This allows Starbug to use the optical communications capability of the AquaFleck, to turn on the docking beacon, and to have one-way communications via the docking rod. This means that when the robots are docked Starbug can communicate with Amour, but not the other way around. This communications limit constrains the scenarios described. Experimental results for the scenarios are presented in the next section.

\subsection{Docking Algorithm}

Docking is a multi-phase process that involves moving to the vicinity of the docking rod on the AquaFleck, aligning with the rod, moving down onto the rod, then locking. The docking algorithm starts with a spiral search phase, during which the robot actively searches for the docking beacon.

The short range target tracking and localization phase uses optical navigation, which has been implemented on Amour. The process begins with the robot sending an optical message to a module resting on the sea floor, instructing it to illuminate its docking beacon. The robot then uses its photodiode array to estimate the direction to the module and this information is used to guide the robot to a position directly above the module. The robot uses its bow thrusters and a PID controller to accomplish this alignment phase.

To join the modules together, one robot aligns itself vertically above the other module (the base module). When all four photodiodes receive a signal of equal strength the robot moves down to land on the base module.

We have tested the docking algorithm for docking between the two robots and docking between the Amour and AquaFlecks. The tests were done in two different pools: one of $1.5 \mathrm{~m}$ depth and another of $5 \mathrm{~m}$ depth. A total of 48 tests were completed: 20 docking operations between Amour and Starbug in the $1.5 \mathrm{~m}$ pool, 8 docking operations between Amour and Starbug in the $5 \mathrm{~m}$ pool, 15 experiments with docking between Amour and AquaFlecks in the $1.5 \mathrm{~m}$ pool and 5 experiments in the $5 \mathrm{~m}$ pool. Each experiment had three phases: spiral search for the beacon, lading on the beacon, and latching. For these experiments, the optical guidance had a range of 2 meters on a 90 degrees cone from the docking rod tip. During these experiments the active robot was perturbed randomly during the spiral search phase and also during the docking phase up to $0.5 \mathrm{~m}$ at a time. The robot docked with Starbug and AquaFleck nodes. 30 of the 48 experiments were successful. The other 18 experiments failed for one of two causes. In 12 experiments the robot failed to complete the latching operation after successfully landing on the rod and this was subsequently found to be due to a docking rod of incorrect length. During 6 experiments the robot experienced loss of optical contact during the landing phase of docking and this was traced to noise in the optical docking sensor. While we have subsequently solved these problems and tested reliable docking, the particular dual robot combination used in this work has not been replicated.

\subsection{Cooperative Motion Control}

Once docked, two AUVs have the ability to travel together combining their resources and ensuring that they reach the destination together. We have developed a cooperative control algorithm that enables Starbug and Amour to maneuver and travel together. We utilize all 9 thrusters of the joined vehicles and Amour's thrusters are effectively slaved to a controller running on Starbug via the unidirectional communications link 


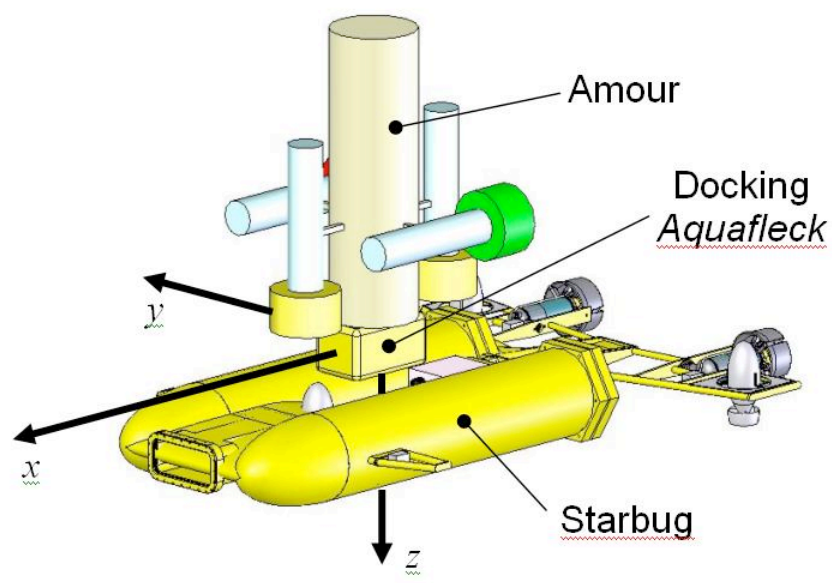

Figure 7: The Cooperative Control Algorithm shows how Amour and Starbug coordinate their resources to accomplish cooperative navigation. Starbug measures horizontal odometry and depth (pressure), closes attitude loops, maintains yaw and sends the actuation demand $\left(F_{x}, F_{Y}, F_{z}, T_{p}\right)$ to Amour.

in the docking rod. Figure 7 describes how the resources are shared. A hydrodynamic model of the combined vehicles is developed and used to synthesize a controller. Experimental results are presented in Section 3.3.

\subsubsection{Modelling of the cooperative docked robots}

In this work, a modified AquaFleck was fitted to the top of Starbug which allowed Amour to physically dock with Starbug and transfer forces from its thrusters to Starbug in the $x, y$ and $z$ directions, including moments about $x$ and $y$, whilst free to spin about the $z$-axis as shown in Figure 8(b).

Generating accurate dynamic models of an AUV is generally a difficult task requiring significant experimental evaluation to determine hydrodynamic coefficients. Empirical results for standard AUV configurations can reduce the amount of experimentation, however, both Starbug and Amour are not standard. For this application the models need only provide a gross estimate of the combined vehicle motion, not high fidelity motion. Therefore, models for both AUVs were developed individually based on the simplified dynamics approach provided by [Nahon, 1996]. As both AUV's have few experimentally determined hydrodynamic coefficients, key parameters are estimated using a series of cylinders and flat plates to represent the vehicle and superimposing the hydrodynamics effects. This assumption typically leads to the greatest source of modelling error, however, given given the relatively slow speeds of the proposed coordinated motion the effect of modified hydrodynamic flow is not as significant.

Models generated for both Starbug and Amour were separately written in such a way that they could be combined for cooperative control. Each vehicle's equations of motion were derived using body fixed coordinates as shown in Figure 8(a) which are located on each vehicle's center of gravity.

The two models include estimates of drag, lift and added mass, as well as weight, buoyancy, thrust and reaction torques. These models were individually tuned to approximate vehicle performance measured and observed during experimentation. The combination of these two models causes some added problems due to the change in mass, inertia, and the center of gravity $(\operatorname{cog})$ locations, $\left(x_{c_{s}}, y_{c_{s}}, z_{c_{s}}\right)$ and $\left(x_{c_{a}}, y_{c_{a}}, z_{c_{a}}\right)$, and buoyancy $(c o b)$ locations, $\left(x_{b_{s}}, y_{b_{s}}, z_{b_{s}}\right)$ and $\left(x_{b_{a}}, y_{b_{a}}, z_{b_{a}}\right)$ for Starbug and Amour respectively. Therefore, the equations of motion and hydrodynamic force estimates of both models were derived using the body fixed 


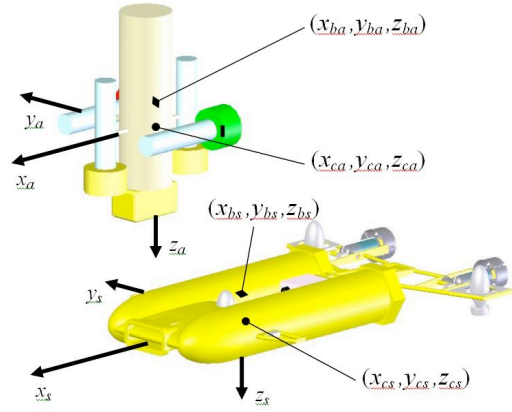

(a)

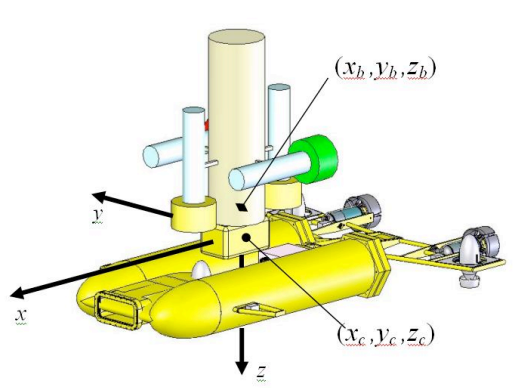

(b)

Figure 8: Coordinate systems for dynamic model development.

coordinate system shown in Figure 8(b) and made functions of the combined robot $\operatorname{cog}\left(x_{c}, y_{c}, z_{c}\right)$ and $c o b$ $\left(x_{b}, y_{b}, z_{b}\right)$ locations so that when combined, they attempt to approximate the total system dynamics. Note that hydrodynamic effects are considered for each model individual, and their change when combined motion is not considered.

The equations of motion for each AUV were written in the form:

$$
\begin{aligned}
\mathbf{F}_{b}+\mathbf{F}_{g}+\mathbf{F}_{c}+\mathbf{F}_{h} & =m \mathbf{a}_{c} \\
\mathbf{M}_{b}+\mathbf{M}_{g}+\mathbf{M}_{c}+\mathbf{M}_{h} & =\mathbf{I}_{c} \dot{\omega}+\omega \times \mathbf{I}_{c} \omega
\end{aligned}
$$

where $\mathbf{F}_{g}, \mathbf{F}_{b}, \mathbf{M}_{g}$ and $\mathbf{M}_{b}$ are the gravity and buoyancy induced forces and moments, and $\mathbf{F}_{c}, \mathbf{F}_{h}, \mathbf{M}_{c}$ and $\mathbf{M}_{h}$ are the control and hydrodynamic forces and moments.

However, when the vehicles are combined as shown in Figure 8(b), Amour is physically locked onto Starbug and transfers forces from its thrusters to Starbug in the $x, y$ and $z$ directions, including moments about $x$ and $y$, whilst free to spin about the $z$-axis. The coordinate system of Figure 8(b) is fixed to Starbug located at the combined vehicle's center of gravity. The total mass, weight and buoyancy of the combined system is the linear addition from both vehicles, and the combined inertia is determined using the parallel axis theorem. The combined center of gravity and buoyancy can be also easily calculated.

In this analysis Amour will provide the control forces for the combined system - this is a function of the one-way communications from Starbug to Amour. Due to the docking mechanism, any pure yaw moment applied to this vehicle results in a rotation about $z$ of Amour only, not Starbug. Therefore, another equation was added to represent the yaw dynamics of Amour which is uncoupled from that of Starbug.

Estimation of hydrodynamic forces was performed by representing each vehicle as a series of $k$ cylinders and flat plates. Each hydrodynamic force and moment for each element is proportional to the square of the linear and angular velocities given by

$$
\begin{aligned}
& \mathbf{v}=(u, v, w) \\
& \omega=(p, q, r)
\end{aligned}
$$

The linear velocities at element $i$ located at $\mathbf{r}_{i}$ relative to the center of gravity is given by

$$
\mathbf{v}_{i}=\mathbf{v}+\omega \times \mathbf{r}_{i}
$$

However the docking mechanism does not prevent rotation about the docking rod and therefore Amour is free to rotation relative to Starbug. The resulting yaw angle between the $x_{s}$ and $x_{a}$ axes $\left(\psi_{s / a}\right)$ in the $x y$ 
plane results in different velocities relative to Amour's coordinate system. Therefore, the velocities need to be transformed to Amour's coordinate system such that $\mathbf{v}_{a i}=\mathbf{T v}$ where

$$
\mathbf{T}=\left[\begin{array}{ccc}
\cos \left(\psi_{s / a}\right) & -\sin \left(\psi_{s / a}\right) & 0 \\
\sin \left(\psi_{s / a}\right) & \cos \left(\psi_{s / a}\right) & 0 \\
0 & 0 & 1
\end{array}\right]
$$

A similar approach is used for the angular velocities. Additionally, as the control forces act in Amour's coordinate system, these too must be transformed to the combined coordinate system for evaluating the system dynamics.

For each AUV, it is assumed that the key mass, inertial and geometric properties are known. It is also assumed that their center of gravity lies on the $x z$ plane. As both AUV's have limited, if any, experimentally determined hydrodynamic coefficients, only low order hydrodynamics and added mass effects are included and approximated using geometry and estimates of drag and lift coefficients with each elements contribution is superimposed. Note that hydrodynamic effects are considered for each model individually, and their change when combined together is not considered. These models were individually tuned to approximate vehicle performance measured and observed during experimentation. The performance of the model in predicting cooperative vehicle motion is presented in Section 3.3.

\subsubsection{Cooperative Control}

Individual autonomous vehicle control has been demonstrated in many different scenarios for both robots. However, coordinated/cooperative control poses a number of challenges due to one-way communications between the vehicles and the physical limitations of the docking mechanism.

The obvious advantage of cooperative control is that with two vehicles coordinating their actions they can achieve motion control that they cannot individually. As described earlier each vehicle is under-actuated, however, in the docking method presented there are unique pose and thrust commands that can achieve fully actuated control of the combination.

In this investigation, it is assumed that once docked Starbug becomes the "master" and uses its onboard sensing capabilities to control Amour's yaw and thrusters to achieve closed-loop depth, roll, pitch and position control of the combination. In addition, Starbug is also able to control its own thrusters to maintain its desired heading, an uncontrolled mode in the combination due to the docking mechanism allowing free rotation about the $z$-axis (see Figure 7).

Due to the uni-directional communications between Starbug and Amour once docked, Starbug does not know the true yaw angle of Amour. Therefore, it is assumed that any thrust or yaw command given to Amour occurs instantaneously, or at least at a time constant that is significantly faster than the dynamics of the combination. This is true in practice as Amour's greater thrust capability, lower rotational inertia and very tight PD control loops mean that the time constant for Amours yaw control $\left(\psi_{a}\right)$ is much greater than that of Starbug's $\left(\psi_{s}\right)$. Hence for control purposes we assume that Amour's yaw angle $\left(\psi_{a}\right)$ is equal to that demanded by Starbug $\left(\psi_{a}^{*}\right)$.

To achieve linear motion control, the forward thrust demand to Amour $\left(u_{x_{a}}^{*}\right)$ is set by proportional control based on position error. However, due to Amour's under-actuated design, to achieve motion in a particular direction, it must first yaw to a desired angle $\left(\psi_{a}^{*}\right)$ then move forward. The demanded yaw angle is set such that Amour points towards the goal position. To avoid rapid yaw motions when close to the goal, a dead-zone is employed and the resulting forward thrust demand is given by

$$
u_{x_{a}}^{*}= \begin{cases}K_{x_{a}}\left(d^{*}-d\right) & \text { if } r>r_{\min } \\ 0 & \text { otherwise }\end{cases}
$$

where $K_{x_{a}}$ is a proportional gain, $d^{*}$ and $d$ are the desired and actual robot positions, $r$ is the Euclidean distance to $d^{*}$ and $r_{\min }$ is the dead-zone boundary. Due to uncontrolled compliance in the docking mechanism when the two robots were connected in addition to a relatively low velocity update rate from the vision system, the dead-zone strategy was found to be more effective in operation than a PD controller. 
Depth control is performed by proportional control based on the measured and desired depth, $z$ and $z^{*}$, such that the up-down thrust demand to Amour is given by

$$
u_{z_{a}}^{*}=K_{z_{a}}\left(z^{*}-z\right)
$$

where $K_{z_{a}}$ is a proportional gain.

The pitching moment caused by the forward thrust of the docked combination is controlled by transforming Starbug's roll and pitch to Amour's local coordinate system such that:

$$
\left\{\begin{array}{l}
\alpha_{a} \\
\phi_{a}
\end{array}\right\}=\left[\begin{array}{cc}
\cos \left(\psi_{a / s}\right) & -\sin \left(\psi_{a / s}\right) \\
\sin \left(\psi_{a / s}\right) & \cos \left(\psi_{a / s}\right)
\end{array}\right]\left\{\begin{array}{c}
\alpha_{s} \\
\phi_{s}
\end{array}\right\}
$$

where

$$
\psi_{a / s}=\psi_{a}^{*}-\psi_{s}
$$

and where $\alpha$ and $\phi$ denote roll and pitch angles respectively, and the subscript indicates the vehicle. From this, the pitching control demand $\left(u_{\phi_{a}}^{*}\right)$ is given by:

$$
u_{\phi_{a}}^{*}=K_{\phi_{a}} \phi_{a}
$$

where $K_{\phi_{a}}$ is a proportional gain.

A large moment can be imposed on the docking module, which tends to bend the rod and distort the lid of the plastic housing. To counteract this, when significant forward thrust is demanded (greater than $\left.u_{x_{\text {Thres }}}\right)$, a counter torque

$$
u_{Q_{a}}^{*}= \begin{cases}K_{v_{x}} u_{x_{a}}^{*}+u_{o f f s e t} & \text { if } u_{x_{a}}^{*}>u_{x_{\text {Thres }}} \\ 0 & \text { otherwise }\end{cases}
$$

was superimposed on Amour's pitching thrusters where $K_{v_{x}}$ is a feed forward gain and $u_{O f f s e t}$ is a prespecified constant. Therefore, the actual up-down thrusts sent to Amours front $\left(u_{F_{a}}\right)$ and back $\left(u_{B_{a}}\right)$ vertical thrusters are a combination of the depth and pitch control demands as well as the bending counter torque such that:

$$
\begin{aligned}
& u_{F_{a}}=\frac{1}{2}\left(u_{z_{a}}^{*}-u_{\phi_{a}}^{*}-u_{Q_{a}}^{*}\right) \\
& u_{B_{a}}=\frac{1}{2}\left(u_{z_{a}}^{*}+u_{\phi_{a}}^{*}+u_{Q_{a}}^{*}\right)
\end{aligned}
$$

The resulting yaw and thruster demands $\left(\psi_{a}^{*}, u_{x_{a}}^{*}, u_{F_{a}}\right.$ and $\left.u_{B_{a}}\right)$ are then sent to Amour via the optical communications link in the docking mechanism.

\subsection{Experiments and results}

Many scenarios for cooperative underwater robotics are possible, and in this work we demonstrate cooperative motion control with two docked AUVs, and a robot rescue scenario. The experiments were conducted in a large indoor pool though both robots have operated in ocean conditions. Both robots are also capable of tetherless operation, though for these experiments it was convenient to use an Ethernet tether on Starbug for experiment control and return of vision data. Multimedia Extension 1 is a collection of short clips illustrating aspects of these experiments.

Cooperative open-loop control This experiment demonstrates the ability of the master robot (Starbug) to command the slave (Amour) whilst docked and evaluates the resulting motion. Figure 9 shows the two AUVs docked with each other during cooperative control tasks.

Figure 10 shows the measured response of the combined vehicle's cooperative motion in which Starbug commanded Amour to yaw 130 degrees then move in its forward direction while Starbug used its on board 


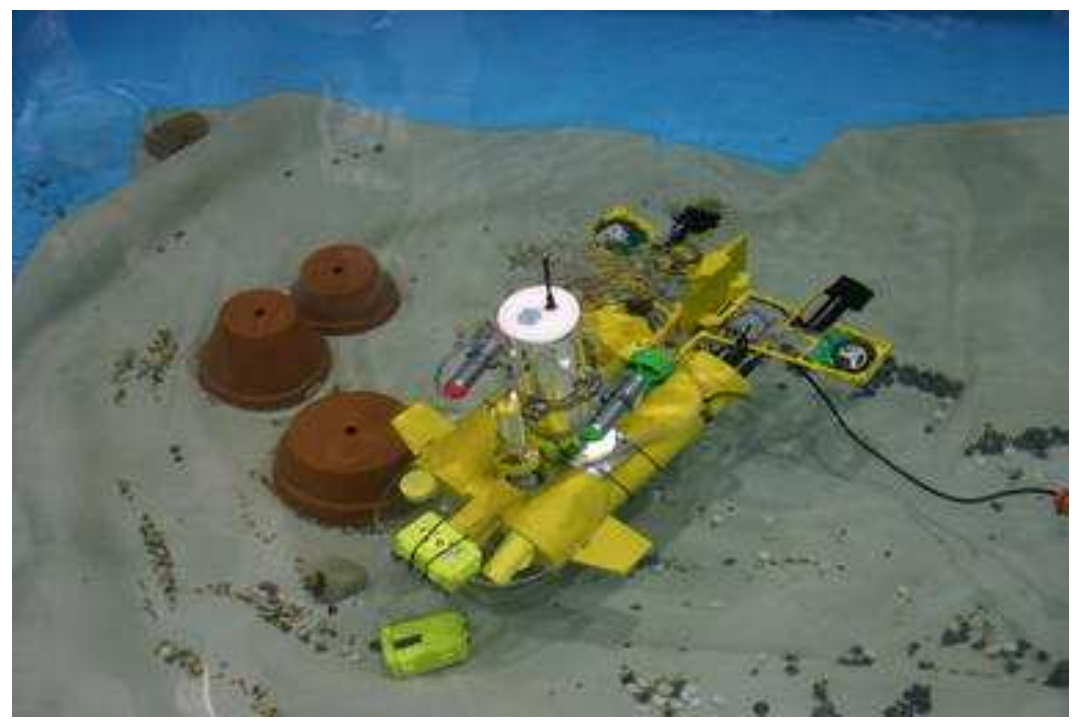

Figure 9: The docked AUVs during cooperative experiments.

pressure sensor to provide vertical control commands to Amour for depth control. In this experiment no pitch or roll control was performed in this experiment (Note that at $t=14 \mathrm{~s}$, the vehicles reached the wall of the test tank, hence the large nose down pitch observed). Additionally, the figure shows the simulated motion provided by the combined model described in Section 3.2.1 for comparison. The simulation model provides a reasonable estimate of the combined vehicle motion and its accuracy is considered suitable for developing further strategies for cooperative control and for anticipating the dynamics of system.

The results in Figure 10 show accurate depth control, however, without any active roll and pitch compensation, the large moment arm from Amour's thrusters causes significant roll and pitch motions of the combined vehicle. Additionally, due to Starbug's large vertical tail and the docking mechanism having no $z$-axis moment control, there is a "weather vaning" effect whereby Starbug aligns its $x$-axis with the direction of travel.

Cooperative vision-based closed-loop position control A series of experiments were conducted to demonstrate cooperative navigation (see Extension 1).

Using the coordinated control algorithm described in Section 3.2.2, an experimental closed-loop position control scenario was set up. Here the Starbug AUV waited on the floor of the test tank while the Amour AUV conducted a search routine to locate and dock with Starbug. Once docked, Starbug used its visionbased position system to control all of Amour's thrusters and follow a desired return path transect. After completing the transect, Starbug commanded Amour to place it back on the floor, undock and return to the surface.

Figure 11 shows the state machine employed for both robots during the coordinated closed-loop control. As communications are uni-directional, the only way Starbug knows that docking has occurred is by waiting until it senses a change in depth. When this is observed, Starbug then commences communications via the AquaFleck to Amour to move to state "DOCKED" at which stage Starbug becomes the master for sensing and control, with Amour the slave.

Figure 12 shows the commanded and measured trajectory from a representative closed-loop cooperative position control experiment. The robot is following straight line segments between way points. In these experiments, not only did Starbug command Amour, but it also used its own thrusters to maintain a commanded yaw angle as well as assist in pitch control. The actual trajectory follows the demanded path reasonably well but with considerable overshoot at the vertices due to shortcomings in the dynamic model 

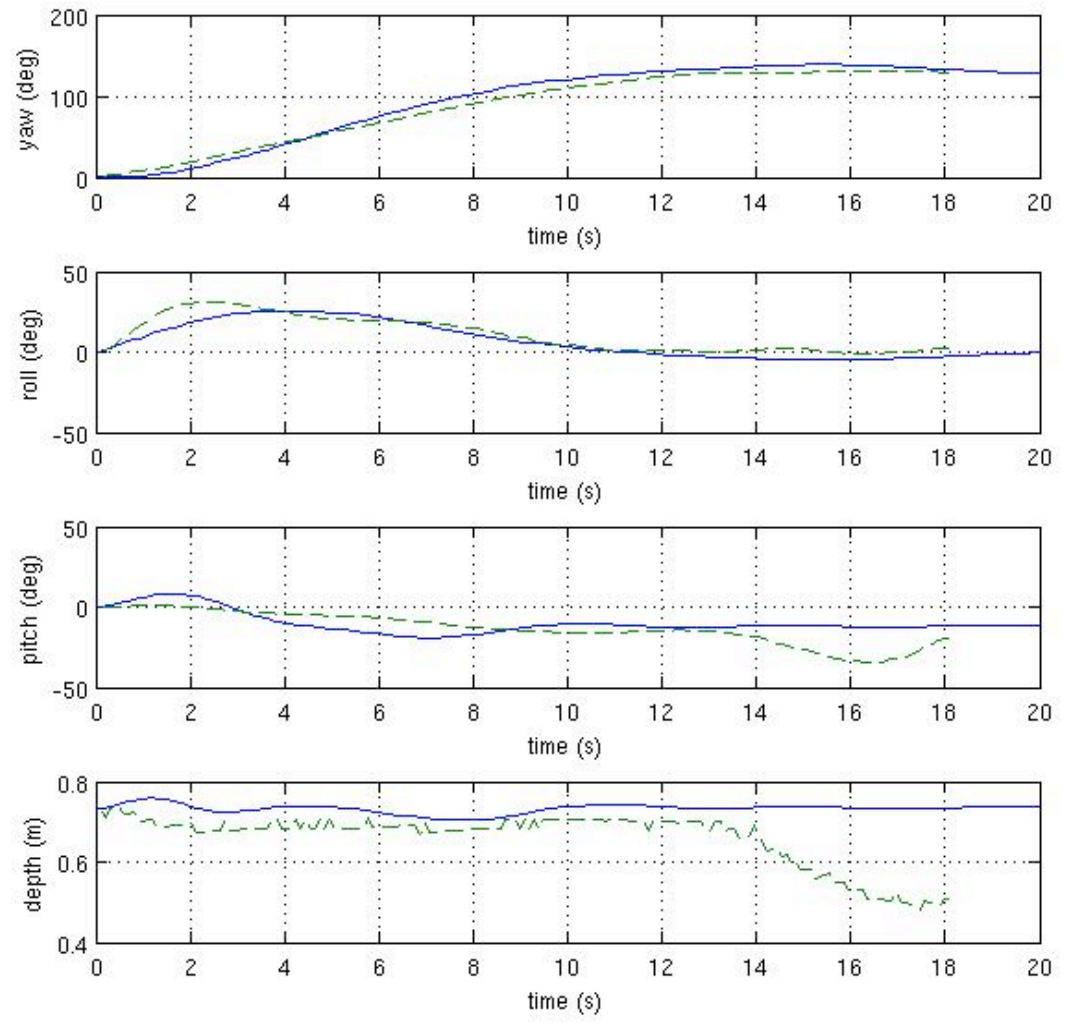

Figure 10: Open-loop cooperative motion. Comparison of simulated (solid line) and observed (dashed line) motion. Yaw is with respect to magnetic north.

and the yaw control dead band (of $0.3 \mathrm{~m}$ ) given in Equation 7. Figure 13 shows a time history of selected pose and Amour thruster commands as measured and demanded by Starbug for Figure 12. A unique outcome of this cooperative control is that Starbug demonstrated pure lateral motion control which was not achievable by Starbug alone (achieved by holding a yaw angle and Amour driving in a perpendicular direction). This demonstrates the ability to extend the capabilities of one robot by coordinated control.

The robots maneuvered together for over 2 hours with consistent performance observed. Figure 14 shows the measured trajectory of three consecutive docking, control, release and search missions illustrating the reliable performance of the coordinated control and docking algorithms. Starbug has only 5 thruster and is not capable of sideways motion, but in the docked configuration it is over actuated and is thus able to move sideways.

Robot rescue In this scenario Starbug is simulating being disabled on the pool floor. In fact, to overcome its slight positive buoyancy it is actively maintaining depth with downward vertical thrust. Amour was able to reliably and autonomously locate Starbug using the beacon-based location and docking algorithms described above. Once docked Armour applies vertical thrust until it reaches the surface. Multiple trials showed the reliability of the docking process. 


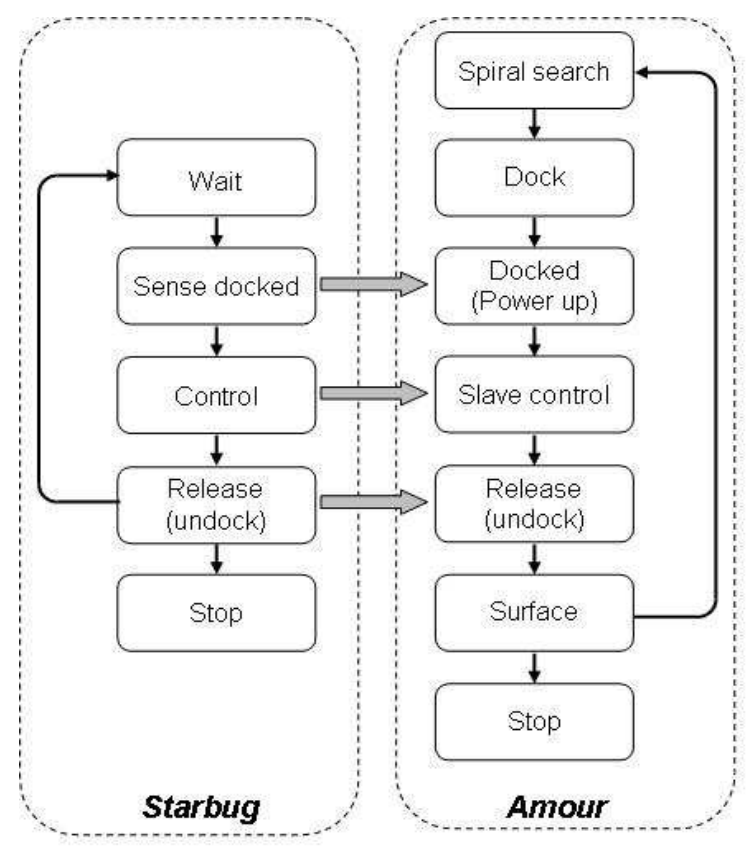

Figure 11: Coordinated control state machine for both Starbug (left) and Amour (right) with uni-directional communications from Starbug to Amour.

\section{Extensions to Goal-Directed Navigation for Data Muling}

Cooperative navigation potentially enables a coupled system of robots to travel long distances together. Once at the destination, the system would separate into components such that each module would be able to function in its task-specific modality. Examples applications that include deploying a sensor network system comprising static and mobile nodes, repositioning a sensor network system, and touring a sensor network system to download the data in the system.

We are continuing our investigations of cooperative underwater robotics by examining how our system of robots and static sensor nodes can work together towards these types of applications. Docking and undocking, when operating in concert with global or local localization systems can be used to deploy and reposition a network. The ability to recognize deployed nodes, an implicit communications, can also be used with the communication capabilities of the system to download the data collected by the nodes without physical contact with the node. In this section we describe our approach to these problems, extending some of the results in [Dunbabin et al., 2006a, Vasilescu et al., 2005].

\subsection{Node recognition}

Each of the robots uses a different approach to locate the AquaFleck nodes. Amour uses active optical beaconing as discussed above, whereas use Starbug uses vision to locate the bright yellow AquaFlecks (see [Vasilescu et al., 2005]). This passive method, where the nodes are identified based on their color, requires no energy expenditure on the part of the node as opposed to active beaconing. It is also easily implemented using Starbug's down-looking color cameras and vision system. A typical Starbug image of a node is shown in Figure 15(a).

Our approach to locating the nodes by color is classical. The color images are converted to normalized red-green chromaticity coordinates and applied to a pre-learnt 2D lookup table, Figure 15(c), which maps the pixels to a binary image, Figure 15(b). Connected region analysis and an area threshold determine 


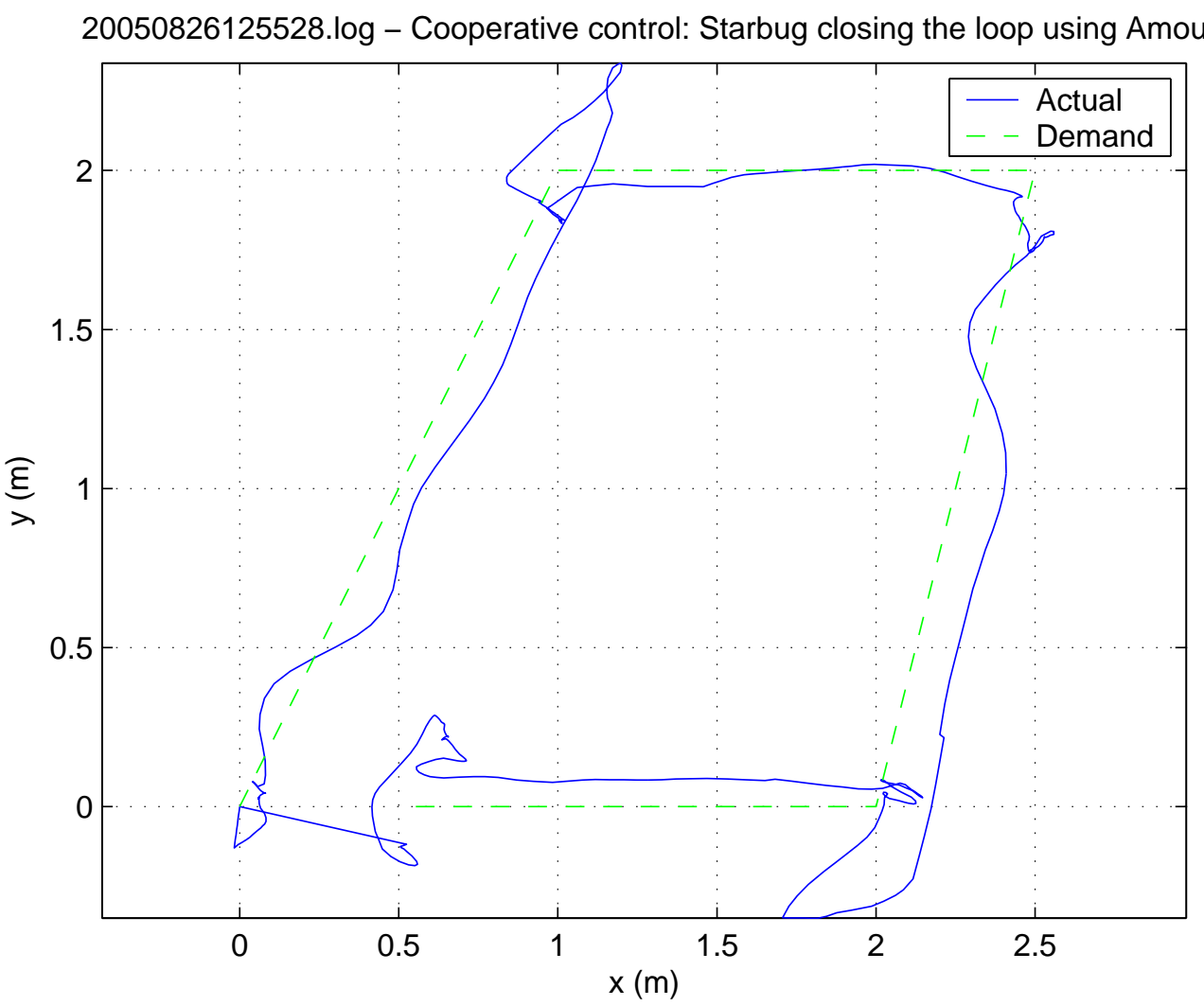

Figure 12: Vision-based closed-loop cooperative motion. The path is clockwise starting at $(0,0)$. Actual and demanded path of the docked AUVs as estimated by Starbug's vision system. Starbug sends thrust and yaw commands to Amour whist the two robots are docked.

the presence of a node. Performing this process with both the left and right camera, we can compute an approximate range based on the centroid disparity, and this allows for adjustment of the area threshold.

Several factors combine to make this a challenging problem [Sattar and Dudek, 2006]. Firstly, a significant area of the top face of the node is not exactly yellow due to lenses, acoustic transducers and sensors. (see Figure 15(a)). Additionally, the preferential absorption of long wavelengths (reds) in water causes marked color change over quite short distances. This filtering occurs in the illumination falling on the node, and also along the path from node to camera. For longer term immersion, obscuration due to biofouling by marine flora and fauna may also occur.

\subsection{Data collection}

Sharing resources between multiple AUVs for navigation and control has been described and demonstrated in Section 3. Extending this to the area of data collection within underwater networks is an interesting and challenging problem. Figure 16 shows how Amour and Starbug can share resources in a goal-directed navigation task where the goal is to tour a set of deployed underwater sensor network nodes and upload the data stored in these nodes. This is called the data muling problem. Figure 16(a) shows a scenario whereby a single robot, for example Starbug, can be used to tour and upload data from deployed nodes using its own propulsion and sensing capabilities. However, as Starbug is not capable of deploying and retrieving network nodes, this concept can be extended to the case of two cooperatively acting robots, Figure 16(b), 

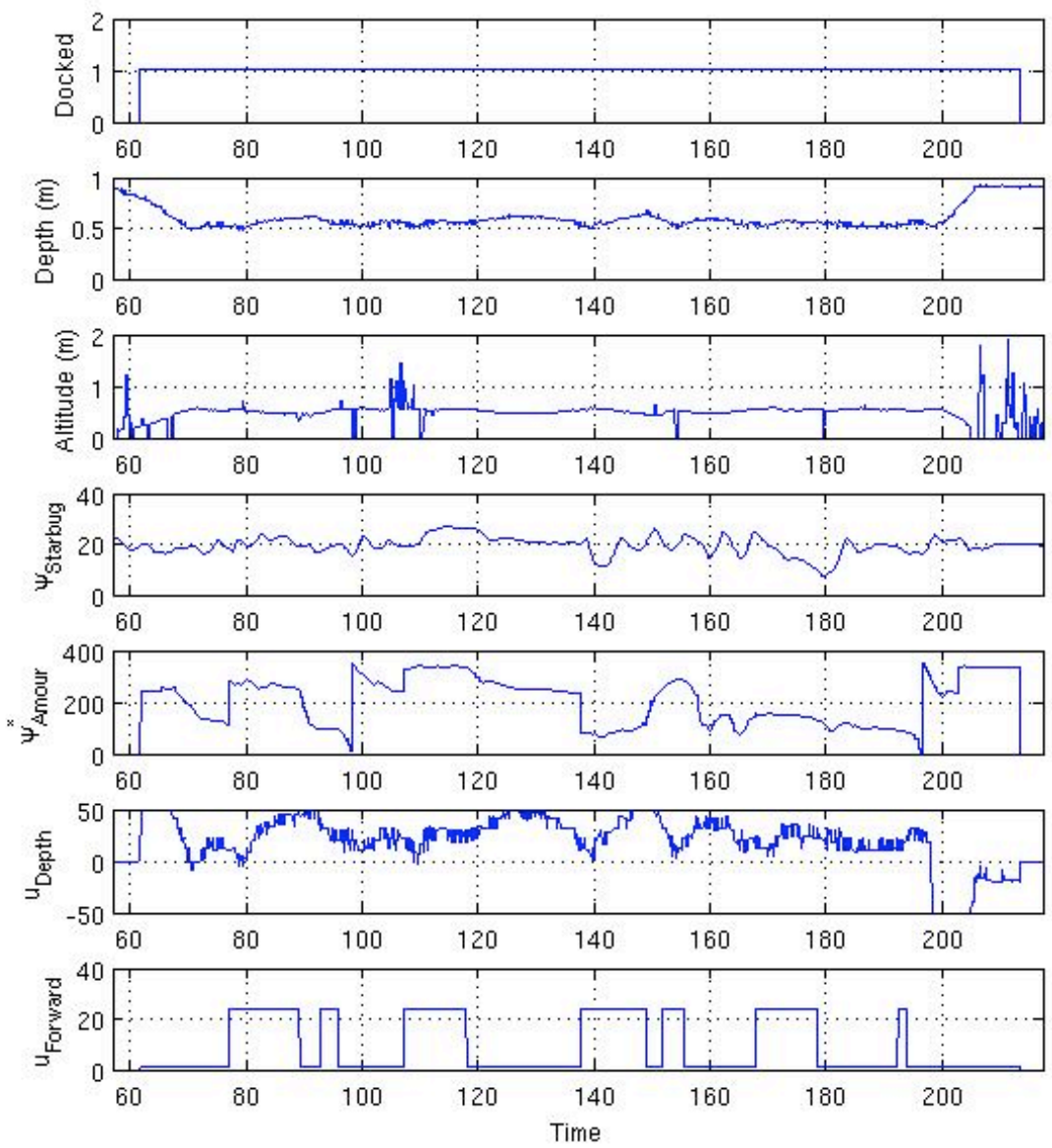

Figure 13: Vision-based closed-loop cooperative motion. Time history of various pose and thruster commands as measured and demanded by Starbug during a cooperation mission. Initially, Starbug is sitting on the bottom of the pool. Amour docks and lifts Starbug at $\mathrm{t}=62 \mathrm{~s}$. Following docking, Starbug provides Amour with control inputs to hold depth at $0.5 \mathrm{~m}$. The vision based altitude is shown as well. Starbug's goal was to hold a constant yaw angle of 20 degrees throughout the mission. Amour's yaw demand is shown to complete path along with the depth input and forward thrust command.

whereby they share resources (propulsion, sensing and node deployment/collection) as described in Section 3 to complete the task.

We have shown [Vasilescu et al., 2005] that we can use the mobile AUVs as data mules to periodically visit and carry data from the AquaFleck nodes back to a base station. The AUV can locate the static nodes using an optical location system. The nodes are mostly in a deep sleep mode and wake every few seconds to determine if they are being optically signalled. This creates a desirable asymmetry in the communications power required, where the AUV which is mobile and rechargeable takes on the energy expensive role.

Data muling, as implemented in these experiments, proceeds in several stages:

1. Compute an AUV route that visits all nodes of interest. 


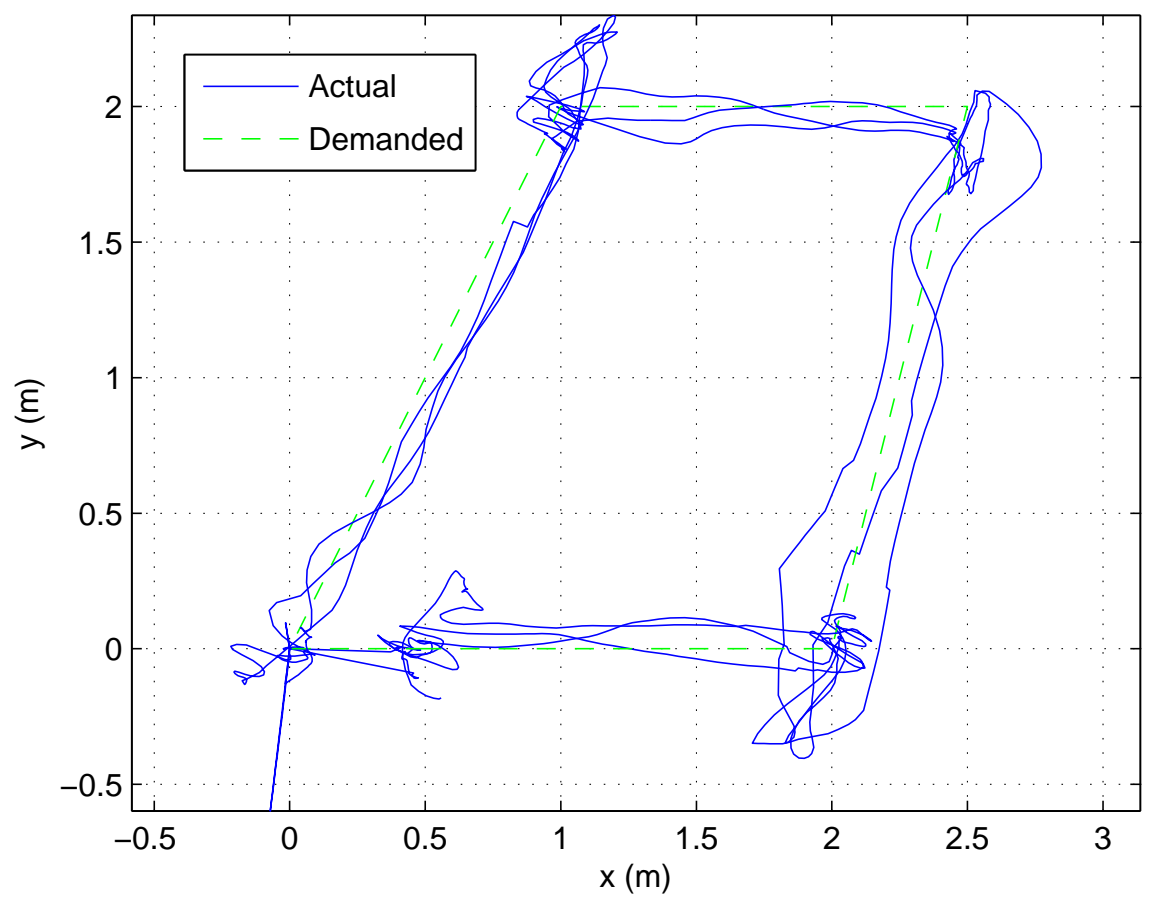

Figure 14: Vision-based closed-loop cooperative motion. Actual and demanded path for three consecutive autonomous docking and cooperative control missions. The path is clockwise starting and ending at $(0,0)$ at which point Amour undocks, moves randomly for $5 \mathrm{~s}$, then commences target search, docking and cooperative navigation and control procedure again (position as estimated by Starbug's vision system).

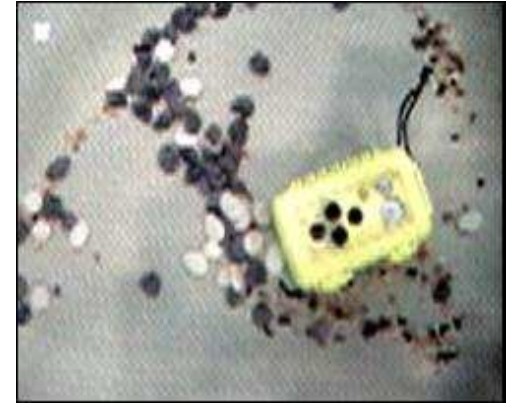

(a) Captured color image

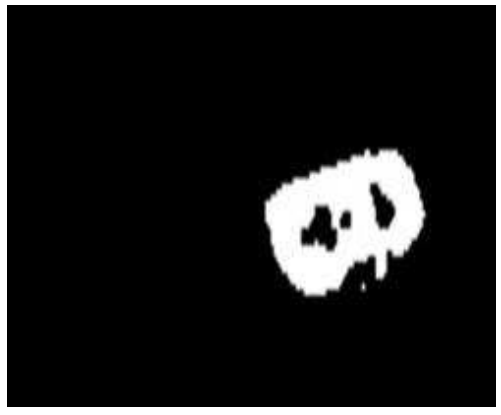

(b) Segmented image

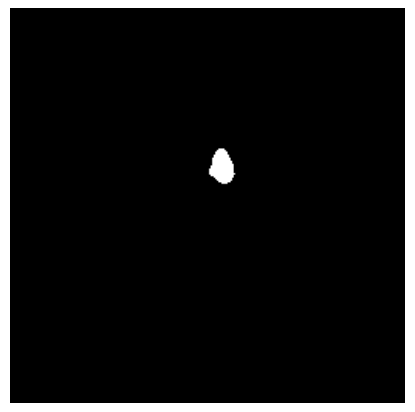

(c) R-G lookup table

Figure 15: Starbug on-board image processing of an AquaFleck on the tank floor using $320 \times 240$ pixel images.

2. Select the next node to be visited from the computed route.

3. Travel to the approximate location of the selected node which requires an ability to navigate and localize underwater

4. Locate the node visually and establish communications. 


\section{Experiment - Starbug}

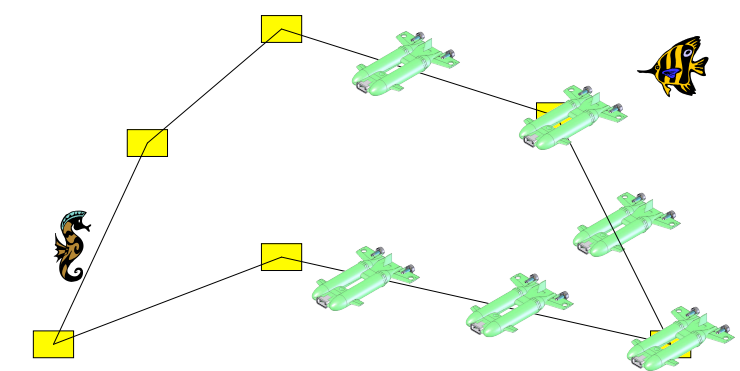

Starbug: perception system, computation, logging, pose

\section{Experiments - Amour side}

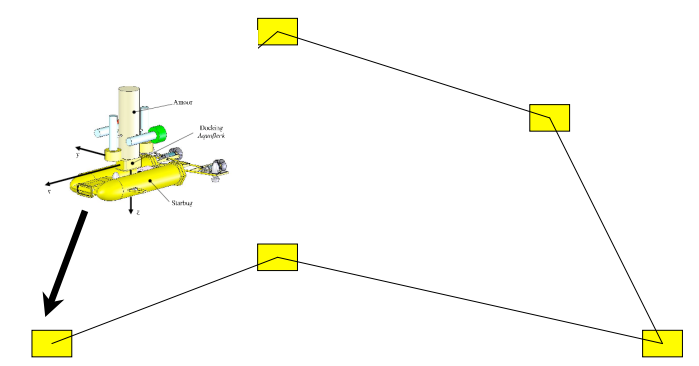

Amour: $(x, y, z)$ actuation system, yaw for $(x, y)$ translation

Figure 16: (Left) Starbug's responsibilities within data muling. (Right) Amour's responsibilities for data muling.

5. Hover while uploading the data.

6. If not the last node, go to (2).

Given an initial starting location the AUV will head toward the first node using either the inboard visionbased position estimation system or dead reckoning navigation based on compass heading and integrated velocity estimated from forward thrust. If the robot does not locate a node within a set distance of where it thinks the node should be, a spiral search is initiated. If a node is detected, the AUV interrogates it for identity and hence its location, and resets its own position estimate. If the located node was not the expected one the AUV replans a path to visit all remaining nodes.

\subsection{Experiments with Sensor Network Tours}

Touring all nodes within an underwater sensor network has been investigated experimentally. A set of nodes were placed on the floor of the test tank and their locations approximately known by the AUV. Using the node identification procedure described in Section 4.1 and the muling strategy of Section 4.2, the Starbug AUV was commanded to visit all nodes and map their location. Although not demonstrated here both robots could have been use to maneuver cooperatively based on the results of Section 3. Furthermore, this scenario can be easily extended to the case whereby Amour with its node docking and deployment capabilities deploys or collects nodes at specific locations being guided cooperatively by Starbug.

Figure 17(a) shows the Starbug's vision-estimated position for the case of zero initial position error. The diamonds indicate the approximate location of the nodes from the a-priori map. As can be seen, the visionbased position estimate coincides well with the map-based node position. Once a node is found, the vision system resets its position estimate to that of the node's map coordinates as shown by the position estimate jumps in the vicinity of the node. Figure 17(b) shows the corrected trajectory using the first node's position to back calculate the AUV's initial start position.

Figure 18 shows the results of the case where the AUV's starting position was $(-1,0)$ rather than the origin that it assumed. Therefore it failed to encounter the first node and after a preset distance limit a search routine was initiated. Figure 18(a) shows the vehicle's estimated position for control. The vision-based position "reset" is clearly seen once the node has been found. Figure 18(b) shows the corrected trajectory using the first found node's position to back calculate the AUV's initial start position. In this instance, the nodes are visited in order.

The final scenario consists of the AUV being started in a position which is not the map origin, and the first node found is not Node 1. Figure 19(a) shows the AUV's position estimate during the search when it believes that it started at the map origin and subsequent resetting of the vehicle's position based on the 


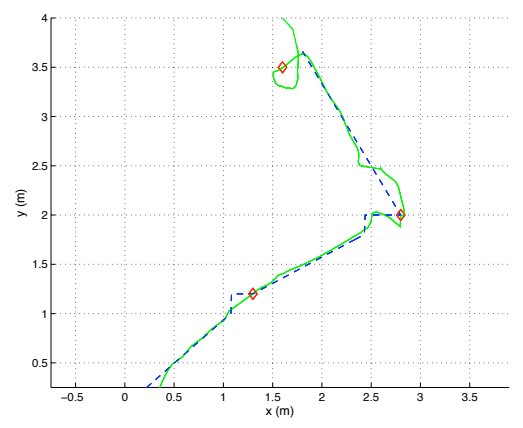

(a) Uncorrected position: actual (solid), demand (dashed)

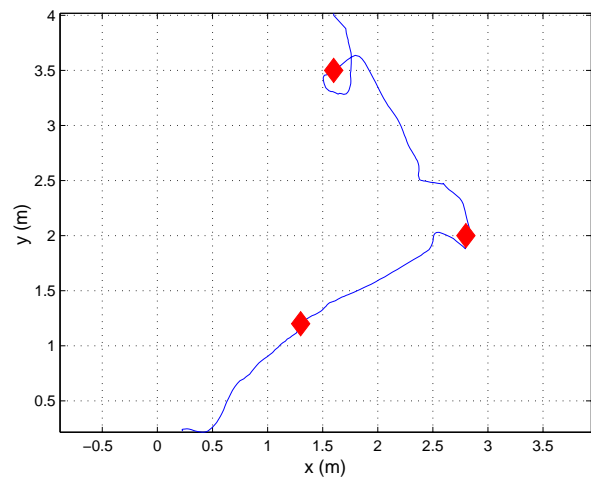

(b) Corrected AUV position estimate

Figure 17: Vision-estimated vehicle position and node position with AUV starting location at map origin. The AUV starts at $(0,0)$ and moves upward in the graph.

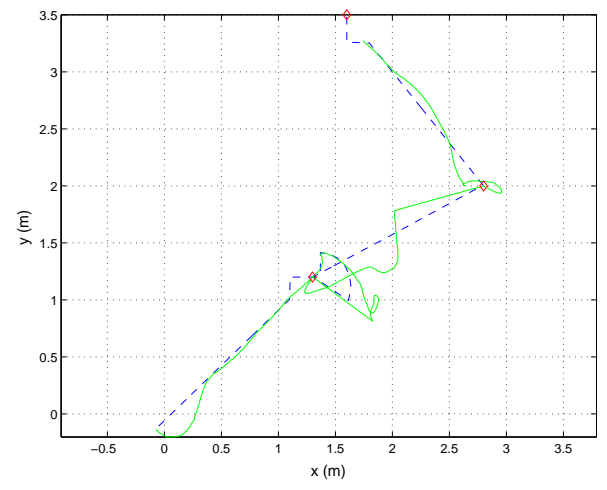

(a) Uncorrected position: actual (solid), demand (dashed)

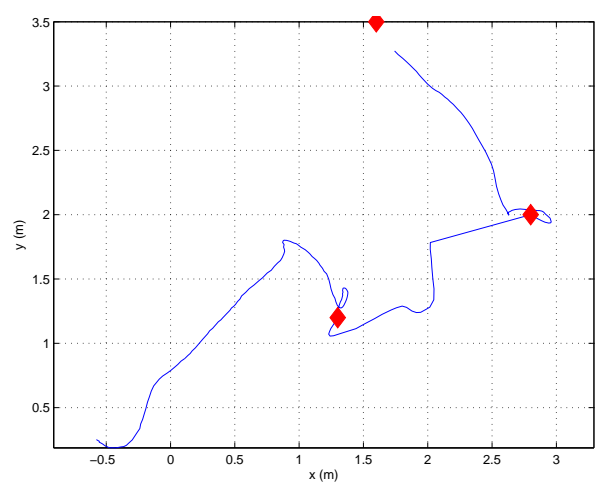

(b) Corrected AUV position estimate

Figure 18: Vision-estimated vehicle position and node position with AUV starting location offset from map origin. The AUV starts at $(0,0)$ and moves upward in the graph.

node identity reported by the first found node. Figure 19(b) shows the corrected trajectory showing the true vehicle position and actual starting location during the experiment. The spiral search can be clearly seen before locating Node 2.

In total, during this experimental campaign Starbug successfully completed over 46 muling missions locating up to 8 nodes per mission in the test tank. These missions involved visiting the nodes in order, out of order, and through spiral searches to locate the nodes in order to test system robustness. These results demonstrate the ability of the networked cooperative action of the both the sensor nodes and AUV to not only autonomously transfer data between each other, but also the ability to allow AUV re-localization and continued mission execution if the AUV becomes initially "lost". In practice path optimization to reduce travel time would be important.

\section{Conclusions}

We have described a heterogeneous system of underwater robots and static sensor network nodes. The elements of this system are useful in their own right, but through cooperation they are able to achieve tasks 


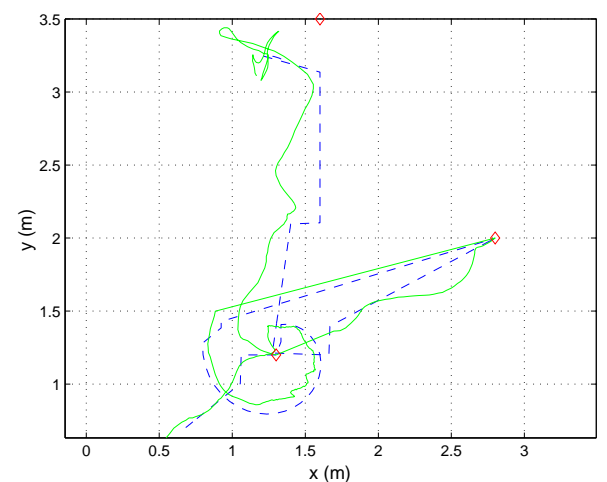

(a) Uncorrected position: actual (solid), demand (dashed)

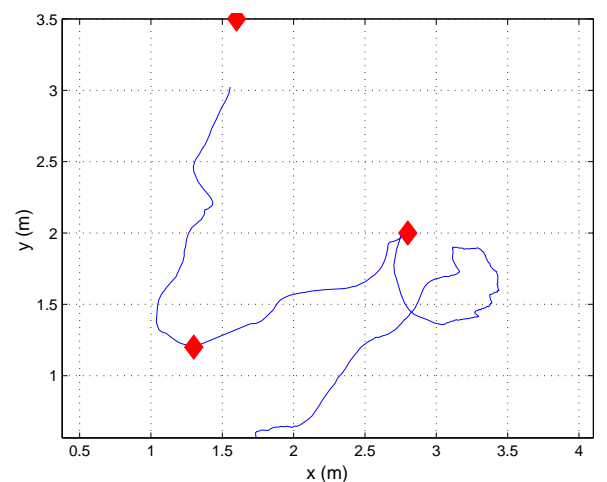

(b) Corrected AUV position estimate

Figure 19: Vision-estimated vehicle position and node position with AUV starting location offset from map origin. Node visitation out of order.

that neither can alone. We believe that achieving long-term underwater monitoring applications requires both specialization to achieve optimal energy performance and maneuverability for each function, but also cooperation and resource sharing amongst these specialist elements.

One class of cooperative tasks includes the robotic deployment, collection and maintenance of sensor nodes, and even their movement to accommodate different sensing needs as events of interest evolve over time. Another class of activities includes physical interaction and coupling between the robots, for example for one robot to rescue another, or for two robots to move together. The coupled robots share not only perceptual capabilities but also actuation and energy. In our experiments two under-actuated robots join to form one over-actuated robot. Given knowledge of the dynamics of the coupled system, we have shown how they can perform controlled motion.

The cooperative primitives that underpin this work are explicit communications by means of long-range acoustic broadcast, and short-range high-speed optical links. There is also implicit communications by which we sense a docking beacon using photodiodes or recognize distinctively colour sensor nodes with a the vision system. Another important primitive is docking, for one robot to another, or one robot to a sensor node.

In this paper we have demonstrated task-level technologies and primitives such as docking, communications and coupled motion over a large number of experiments. The very different AUVs are able to dock and navigate coupled together, and also to achieve goal direction navigation with both implicit and explicit communications with sensor nodes. We have described the control algorithm and experimental results obtained in several test tanks. We believe that cooperation is a key ingredient in creating underwater networked systems of robots and sensors capable of long-term operation. This paper presents some first steps in this direction.

\section{Acknowledgements}

We are grateful to NSF, Intel, and the ONR PLUSNet project for partial funding. We thank Keith Kotay and to the entire team at CSIRO for the technical and moral support provided during a period of intense experimentation; Pavan Sikka for the optical communication stack; Poe Chen for the interface between Flecks and Starbug and support for data muling; and Leslie Overs for electronics support.

\section{Index to Multimedia Extensions}

The multimedia extension page is found at http://www.ijrr.org. 
Table of Multimedia Extensions

Extension Type Description

1 Video Cooperative underwater motion.

\section{References}

[Ayers et al., 2000] Ayers, J., Wilbur, C., and Olcott, C. (2000). Lamprey robots. In Proceedings of the International Symposium on Aqua Biomechanisms.

[Bellingham et al., 1994] Bellingham, J. G., Goudey, C. A., Consi, T. R., Bales, J. W., Atwood, D. K., Leonard, J. J., and Chryssostomidis, C. (1994). A second generation survey AUV. In IEEE Conference on Autonomous Underwater Vehicles, pages 148-155, Cambridge, MA, USA.

[Bokser et al., 2004] Bokser, V., Oberg, C., Sukhatme, G. S., and Requicha, A. A. (2004). A small submarine robot for experiments in underwater sensor networks. In International Federation of Automatic Control Symposium on Intelligent Autonomous Vehicles.

[Choi et al., 2003] Choi, H. T., Hanai, A., Choi, S., and Yuh, J. (2003). Development of an underwater robot, ODIN-III. In Proc. of the International Conference on Intelligent Robot Systems (IROS), pages 836-841.

[Corke et al., 2007] Corke, P., Detweiler, C., Dunbabin, M., Hamilton, M., Vasilescu, I., and Rus, D. (2007). Experiments with underwater localization and tracking. In IEEE ICRA, pages 4556-4561, Rome, Italy.

[Corke et al., 2004a] Corke, P., Hrabar, S., Peterson, R., Rus, D., Saripalli, S., and Sukhatme, G. (2004a). Autonomous deployment and repair of a sensor network using an unmanned aerial vehicle. In Int. Conf. Robotics and Automation, pages 3602-3608, New Orleans.

[Corke et al., 2004b] Corke, P., Hrabar, S., Peterson, R., Rus, D., Saripalli, S., and Sukhatme, G. (2004b). Deployment and connectivity repair of a sensor network with a flying robot. In Preprints Int.Symp. Experimental Robotics, pages 333-343, Singapore.

[Cowen et al., 1997] Cowen, S., Briest, S., and Dombrowski, J. (1997). Underwater docking of autonomous undersea vehicles using optical terminal guidance. In Oceans97, MTS/IEE Conference, pages 1143-1147.

[Detweiler et al., 2006] Detweiler, C., Leonard, J., Rus, D., and Teller, S. (2006). Passive mobile robot localization within a fixed beacon field. In Proceedings of the International Workshop on the Algorithmic Foundations of Robotics, New York, New York. Springer-Verlag.

[Dunbabin et al., 2004] Dunbabin, M., Corke, P., and Buskey, G. (2004). Low-cost vision-based AUV guidance system for reef navigation. In Proceedings of the 2004 International Conference on Robotics and Automation, pages 7-12, New Orleans.

[Dunbabin et al., 2005] Dunbabin, M., Roberts, J., Usher, K., Winstanley, G., and Corke, P. (2005). A hybrid AUV design for shallow water reef navigation. In Proceedings of the 2005 International Conference on Robotics and Automation, pages 2105-2110, Barcelona.

[Dunbabin et al., 2006a] Dunbabin, M., Vasilescu, I., Corke, P., and Rus, D. (2006a). Data muling over an underwater sensor network with an underwater robot. In Proceedings of the IEEE International Conference on Robotics \&3 Automation ICRA, pages 2091-2098, Orlando, FL.

[Dunbabin et al., 2006b] Dunbabin, M., Vasilescu, I., Corke, P., and Rus, D. (2006b). Experiments with cooperative underwater networked robots. In International Symposium on Robotics Research.

[Eriksen et al., 2001] Eriksen, C. C., Osse, T. J., Light, R. D., Wen, T., Lehman, T. W., and Sabin, P. L. (2001). Seaglider: A long-range autonomous underwater vehicle for oceanographic research. IEEE Journal of Oceanic Engineering, 26(4):424-436. 
[Fiorelli et al., 2004] Fiorelli, E., Leonard, N. E., Bhatta, P., Paley, D., Bachmayer, R., and Fratantoni, D. M. (2004). Multi-AUV control and adaptive sampling in Monterey Bay. In Proceedings of the IEEE Autonomous Underwater Vehicles: Workshop on Multiple AUV Operations, pages 134-147, Sebasco, ME, USA.

[Hanai et al., 2004] Hanai, A., Rosa, K., Choi, S., and Yuh, J. (2004). Experimental analysis and implementation of redundant thrusters for underwater robots. In Proc. of the International Conference on Intelligent Robot Systems (IROS), volume 2, pages 1109-1114.

[Huster and Rock, 2003] Huster, A. and Rock, S. (2003). Relative position sensing by fusing monocular vision and inertial rate sensors. In Proc. 11th International Conference on Advanced Robotics, pages 1562-1567, Coimbra, Portugal.

[Kalantar and Zimmer, 2004] Kalantar, S. and Zimmer, U. R. (2004). Contour shaped formation control for autonomous underwater vehicles using canonical shape descriptors and deformable models. In Proceedings of the IEEE International Conference on Marine Technology and Ocean Science, Kobe, Japan.

[Leonard et al., 1998] Leonard, J. J., Bennett, A. A., Smith, C. M., and Feder, H. J. S. (1998). Autonomous underwater vehicle navigation. Memorandum 98-1, MIT Marine Robotics Laboratory, Cambridge, MA, USA.

[McIsaac and Ostrowski, 1999] McIsaac, K. and Ostrowski, J. (1999). A geometric approach to anguilliform locomotion: Modelling of an underwater eel robot. In Proceedings of the IEEE Conference of Robotics and Automation, pages 2843-2848.

[Moore et al., 2004] Moore, D., Leonard, J., Rus, D., and Teller, S. (2004). Robust distributed network localization with noisy range measurements. In ACM SenSys, Baltimore, MD.

[Nahon, 1996] Nahon, M. (1996). A simplified dynamics model for autonomous underwater vehicles. In Proceedings AUV'96, pages 373-379.

[Park et al., 2007] Park, J.-Y., Jun, B.-H., Lee, P.-M., Lee, F.-Y., and ho Oh, J. (2007). Experiment on underwater docking of an autonomous underwater vehicle 'ISiMI' using optical terminal guidance. In OCEANS 2007 - Europe, pages 1-6.

[Pizarro et al., 2004] Pizarro, O., Eustice, R., and Singh, H. (2004). Large area 3D reconstructions from underwater surveys. In OCEANS '04. MTTS/IEEE TECHNO-OCEAN '04, volume 2, pages 678-687.

[Sattar and Dudek, 2006] Sattar, J. and Dudek, G. (2006). On the performance of color tracking algorithms for underwater robots under varying lighting and visibility. In Int. Conf. Robotics and Automation, pages $3550-3555$.

[Sikka et al., 2004] Sikka, P., Corke, P., and Overs, L. (2004). Wireless sensor devices for animal tracking and control. In Proc. First IEEE Workshop on Embedded Networked Sensors, pages 446-454, Tampa, Florida.

[Singh et al., 1997] Singh, H., Bowen, M., Hover, F., LeBas, P., and Yoerger, D. (1997). Intelligent Docking for an Autonomous Ocean Sampling Network. In Oceans97, MTS/IEE Conference, pages 1136-1131, Halifax.

[Vasilescu et al., 2005] Vasilescu, I., Kotay, K., Rus, D., Corke, P., and Dunbabin, M. (2005). Data collection, storage and retrieval with an underwater sensor network. In ACM Sensys, pages 154-165.

[Walter et al., 2007] Walter, M. R., Eustice, R. M., and Leonard, J. J. (2007). Exactly sparse extended information filters for feature-based SLAM. Intl. J. Robotics Research, 26(4):335-359. 
[Wettergreen et al., 1999] Wettergreen, D., Gaskett, C., and Zelinsky, A. (1999). Autonomous control and guidance for an underwater robotic vehicle. In Proceedings of the International Conference on Field and Service Robotics, Pittsburgh, USA.

[Whitcomb, 2000] Whitcomb, L. L. (2000). Underwater robotics: Out of the research laboratory and into the field. In Proceedings of the IEEE International Conference on Robotics and Automation, pages 709-716.

[Whitcomb et al., 1999] Whitcomb, L. L., Yoerger, D. R., and Singh, H. (1999). Combined Doppler/LBL based navigation of underwater vehicles. In Proceedings of the 11th International Symposium on Unmanned Untethered Submersible Technology, Durham, NH, USA.

[Yuh, 2000] Yuh, J. (2000). Design and control of autonomous underwater robots: A survey. Autonomous Robots, 8(1):7-24. 